\title{
EPOS2020: development strategy and goals for the latest European Position Paper on Rhinosinusitis*
}

Wytske Fokkens ${ }^{1}$, Martin Desrosiers ${ }^{2}$, Richard Harvey ${ }^{3}$, Claire Hopkins ${ }^{4}$, Joaquim Mullol ${ }^{5}$, Carl Philpott ${ }^{6,7}$, Isam Alobid ${ }^{8}$, Wilma Terezinha AnselmoLima ${ }^{9}$, Claus Bachert ${ }^{10,11}$, Fuad Baroody ${ }^{12}$, Manuel Bernal-Sprekelsen ${ }^{13}$, Christian von Buchwald ${ }^{14}$, Anders Cervin ${ }^{15}$, Noam Cohen ${ }^{16}$, Jannis Constantinidis ${ }^{17}$, Ludovic De Gabory ${ }^{18}$, Richard Douglas ${ }^{19}$, Philippe Gevaert ${ }^{20}$, Anita Hafner ${ }^{21}$, Peter Hellings 1,10,22, Guy Joos ${ }^{23}$, Livije Kalogjera ${ }^{24}$, Rob Kernn ${ }^{25}$, Andrew Knill26, Janwillem Kocks ${ }^{27}$, Basile Nicolas Landis ${ }^{28}$, Jacqueline Limpens ${ }^{29}$, Sarah Lebeer $^{30}$, Olga Lourenco ${ }^{31}$, Paolo Maria Matricardi32, Cem Meco ${ }^{33,34}$, Liam O'Mahony ${ }^{35}$, Sietze Reitsma', Dermot Ryan ${ }^{36,37}$, Rodney Schlosser ${ }^{38}$, Brent Senior ${ }^{39}$, Thijs Teeling ${ }^{40}$, Peter Valentin Tomazic ${ }^{41}$, Sanna Toppila-Salmi ${ }^{42}$, De Yun Wang ${ }^{43}$, Dehui Wang ${ }^{44}$, Luo Zhang ${ }^{45}$, Valerie Lund ${ }^{46}$
Rhinology 57: 3, 162 - 169, 2019

https://doi.org/10.4193/Rhin 19.080

*Received for publication:

February 6, 2019

Accepted: February 7, 2019

${ }^{1}$ Department of Otorhinolaryngology, Amsterdam University Medical Centres, location AMC, Amsterdam, The Netherlands

${ }^{2}$ Department of ORL-HNS, Université de Montréal, Montreal, Canada

${ }^{3}$ Rhinology and Skull Base Department, Applied Medical Research Centre, UNSW (Conjoint) and Macquarie University (Clinical), Sydney, Australia

${ }^{4}$ Ear, Nose and Throat Department, Guys and St. Thomas' Hospital, London, United Kingdom

${ }^{5}$ Rhinology Unit and Smell Clinic, ENT Department, Hospital Clínic, IDIBAPS, Universitat de Barcelona, CIBERES, Barcelona, Catalonia, Spain

${ }^{6}$ Department of Medicine, Norwich Medical School, University of East Anglia, Norwich, UK

${ }^{7}$ ENT Department, James Paget University Hospital, Great Yarmouth, UK

${ }^{8}$ Rhinology and Skull Base Unit, ENT Department, Hospital Clínic de Barcelona, Universidad de Barcelona, August Pi i Sunyer Biomedical Research Institute, Barcelona, Spain

${ }^{9}$ Division of Otorhinolaryngology, Department of Ophthalmology, Otorhinolaryngology, Head and Neck Surgery, Ribeirao Preto Medical SchoolUniversity of Sao Paulo, Sao Paulo, Brazil

${ }^{10}$ Upper Airways Research Laboratory and ENT Department, University Hospital Ghent, Ghent, Belgium

${ }^{11}$ Division of ENT Diseases, CLINTEC, Karolinska Institute, University of Stockholm, Stockholm, Sweden

${ }^{12}$ Department of Otolaryngology-Head and Neck Surgery, The University of Chicago Medicine and the Comer Children's Hospital, Chicago, IL, USA

${ }^{13}$ ENT Department Hospital Quironsalud, University of Valencia, Valencia, Spain

${ }^{14}$ Department of Otolaryngology, Head and Neck Surgery and Audiology, Rigshospitalet, Copenhagen University, Hospital, Copenhagen, Denmark ${ }^{15}$ Department of Otolaryngology, Head and Neck Surgery, Royal Brisbane and Women's Hospital; Faculty of Medicine, University of Queensland, Brisbane, Australia

${ }^{16}$ Department of Otorhinolaryngology - Head and Neck Surgery, Perelman School of Medicine at The University of Pennsylvania, Philadelphia, PA, USA

17 1st Department of ORL, Head and Neck Surgery, Aristotle University, AHEPA Hospital, Thessaloniki, Greece

${ }^{18}$ Rhinology and Plastic Surgery Unit, Otorhinolaryngology, Head and Neck Surgery and Pediatric ENT Department, CHU de Bordeaux, Hôpital Pellegrin, Centre F-X Michelet, Bordeaux, France

${ }^{19}$ Department of Surgery, The University of Auckland, Auckland, New Zealand

${ }^{20}$ Department of Otorhinolaryngology, Ghent University, Ghent, Belgium

${ }^{21}$ University of Zagreb Faculty of Pharmacy and Biochemistry, Zagreb, Croatia

${ }^{22}$ Department of Otorhinolaryngology, Head and Neck Surgery, University Hospitals Leuven, KU Leuven, Leuven, Belgium

${ }^{23}$ Department of Respiratory Medicine, Ghent University Hospital, Ghent, Belgium

${ }^{24}$ ENT Department, Zagreb School of Medicine, University Hospital Center "Sestre milosrdnice", Zagreb, Croatia 
${ }^{25}$ Department of Otolaryngology - Head and Neck Surgery, Northwestern University, Feinberg School of Medicine, Chicago, IL, USA

${ }^{26}$ Patient representative, Opuscomms, London, UK

${ }^{27}$ Department of Inhalation Medicine, Observational Pragmatic Research Institute, Singapore

${ }^{28}$ Rhinology-Olfactology Unit, Otorhinolaryngology Department, University Hospital of Geneva, Geneva, Switzerland

${ }^{29}$ Medical Information Specialist, Medical Library, Amsterdam University Medical Centres, location AMC, Amsterdam, The Netherlands

${ }^{30}$ Department of Bioscience Engineering, University of Antwerp, Antwerp, Belgium

${ }^{31}$ FCS - UBI Faculty of Health Sciences, University of Beira Interior, Covilhã, Portugal

32 Department of Pediatric Pneumology and Immunology, Charité - Universitätsmedizin Berlin, Berlin, Germany

${ }^{33}$ Department of Otorhinolaryngology, Head and Neck Surgery, Ankara University, Ankara, Turkey

${ }^{34}$ Department of Otorhinolaryngology, Head and Neck Surgery, Salzburg Paracelsus Medical University, Salzburg, Austria

${ }^{35}$ Departments of Medicine and Microbiology, APC Microbiome Ireland, National University of Ireland, Cork, Ireland

${ }^{36}$ Allergy and Respiratory Research Group, Usher Institute of Population Health Sciences and Informatics, University of Edinburgh, Edinburgh, UK

${ }^{37}$ Optimum Patient Care, Cambridgeshire, UK

${ }^{38}$ Department of Otolaryngology - Head and Neck Surgery, Medical University of South Carolina, Charleston, USA

${ }^{39}$ UNC Otolaryngology / Head and Neck Surgery, Division of Rhinology, Allergy, and Endoscopic Skull Base Surgery and Department of Neurosurgery, University of North Carolina School of Medicine, Chapel Hill, NC, USA

${ }^{40}$ Patient representative, Task Force Healthcare, WTC Den Haag, The Netherlands

${ }^{41}$ Department of Otorhinolaryngology, Head and Neck Surgery, Medical University of Graz, Graz, Austria

${ }^{42}$ Skin and Allergy Hospital, Helsinki University Hospital and University of Helsinki, Helsinki, Finland

${ }^{43}$ Department of Otolaryngology, Yong Loo Lin School of Medicine, National University of Singapore, Singapore

${ }^{44}$ Rhinology Division, ENT Department; Eye and ENT Hospital, Fudan University, Shanghai, China

${ }^{45}$ Department of Otolaryngology Head and Neck Surgery, Beijing TongRen Hospital, Beijing, China

${ }^{46}$ Royal National Throat, Nose and Ear Hospital, UCLH, London, UK

\section{Abstract}

Background: The European Position Papers on Rhinosinusitis from 2005, 2007 and 2012 have had a measurable impact on the way this common, yet burdensome condition is diagnosed and treated around the world. EPOS2020 will be the latest iteration of the guideline, addressing new stakeholders and target users, presenting a summary of the latest literature and evolving treatment modalities, and formulating clear recommendations based on all available evidence.

Methodology: Based on the AGREE II framework, this article demonstrates how the EPOS2020 steering group will address six key areas to ensure consistency in quality and presentation of information in the latest rhinosinusitis clinical practice guideline: scope and purpose; stakeholder involvement; rigour of development; clarity of presentation; recommendations and applicability; editorial independence.

Results: By analysing the guidance from AGREE II we have formulated a detailed development strategy for EPOS2020. We identify new stakeholders and target users and ratify the importance of patient involvement in the latest EPOS guideline. New and expanded areas of research to be addressed are highlighted. And we confirm our intention to use mixed methodologies, combining evidence-based medicine with expert opinion to achieve clear, inclusive recommendations. We also introduce new concepts for dissemination of the guideline, making use of the Internet and social media to improve accessibility.

Conclusion: This article is an introduction to the EPOS2020 project, and presents the key goals, core stakeholders, planned methodology and dissemination strategies for the latest version of this influential guideline.

Key words: rhinosinusitis, EPOS, methodology, recommendations, stakeholders, patients, nasal polyps, upper respiratory tract infection, asthma 


\section{Introduction}

Rhinosinusitis is a common condition in most of the world, leading to a significant burden on society in terms of healthcare consumption and productivity loss ${ }^{(1-4)}$.

Acute rhinosinusitis (ARS) has a 1-year prevalence of 6-15\% and is usually the consequence of a viral common cold. ARS is usually a self-limiting disease but serious complications leading to life threatening situations and even death have been described ${ }^{(5)}$. It is one of the most common reasons for prescription of antibiotics and proper management is extremely pertinent in the context of the global crisis of antibiotics ${ }^{(6)}$. Chronic rhinosinusitis (CRS) is a significant health problem and affects $5-12 \%$ of the general population ${ }^{(1,2,7,8)}$. CRS can present with (CRSwNP) or without nasal polyps (CRSsNP). The first group in particular often needs recurrent surgical procedures and systemic treatment with corticosteroids and is associated with severe asthma (9-14). The European Position Paper on Rhinosinusitis (EPOS 2020) will be the latest in the EPOS series of guidelines on rhinosinusitis from an international cohort of experts in the field.

The first European Position Paper on Rhinosinusitis and Nasal Polyps (EP3OS) ${ }^{(15,16)}$ was published in 2005 , and was soon followed by EP3OS $2007^{(17,18)}$ in response to the first position paper's extremely positive reception and the growing body of research in this area.

The latest European Position Paper on Rhinosinusitis, EPOS 2012 ${ }^{(19,20)}$ was hugely successful and widely cited worldwide. It delivered a comprehensive review of the key topics in an accessible format with clear research priorities.

EPOS has inspired researchers to use standard definitions of disease, study the epidemiology and management of ARS and CRS, reduce unnecessary (mainly antibiotic) treatment and form new collaborations to answer unmet research questions ${ }^{(9,21-26)}$. EPOS2020 will build on this success. We plan to utilize a combination of methodologies to expand on the existing research base and explore areas not covered in the previous guidelines. This article outlines our development strategies in order to achieve these goals based on the AGREE II framework (27), a tool which aims to ensure consistency in quality of clinical practice guidelines, provide a methodological strategy for the development of guidelines and clarify what and how information ought to be reported in guidelines.

\section{Scope and purpose}

The core objective of the EPOS2020 guideline is to provide real, practical, collaborative management strategies for rhinosinusitis based on a combination of evidence based and clinically relevant research. We aim to build on the success of previous EPOS position papers, in particular EPOS2012, which was widely acclaimed for its presentation of science translated into clinical care and treatment of disease. EPOS2012 can also be credited with the sharp rise in understanding of separate endotypes in
Table 1. New and expanded areas of research for EPOS2020.

Developments in understanding the pathophysiology of rhinosinusitis, their translation into clinics (endotyping), with emphasis on the consequences for the use of biologicals.

Role of biologicals cost effectiveness / cost benefit analysis, efficacy versus safety

Surgical management of both acute and chronic rhinosinusitis (adult and paediatric) with finer details and new technologies; extent of surgery; revision surgery.

Genome / epigenome results for rhinosinusitis

Precision medicine in rhinosinusitis

Emergencies in rhinosinusitis

Systemic diseases affecting the nose and paranasal sinuses

Importance of loss of smell in diagnosis / severity / control of disease

Sleep problems in patients with CRS

Integrated care pathways / collaboration and consultation with other specialties

The 'difficult to treat patient'

Molecular allergology in the diagnosis of rhinosinusitis

Mobile Health technology in the management of rhinosinusitis

CRS and the relationship to different phenotypes. This knowledge will lead to significant adjustments in the management of CRS, especially in CRSWNP ${ }^{(28,29)}$.

All topics from EPOS2012 will be revised and updated based on the latest research and evidence. Areas that EPOS2020 will explore in more detail than in previous guidelines are summarised in Table 1. In particular, evolving treatment modalities especially with biologicals, need reevaluation in terms of current treatment algorithms ${ }^{(30,31)}$.

The EPOS 2020 guideline will apply to the adult and paediatric patient population with ARS (viral / common cold, post-viral, bacterial), CRSsNP and CRSwNP. It will furthermore be applicable to patients with associated conditions, such as allergic rhinitis, asthma, bronchiectasis, chronic obstructive pulmonary disease (COPD), severe upper airway disease and rare diseases including primary ciliary dyskinesia (PCD), cystic fibrosis, vasculitis and immune deficiencies. EPOS2020 will also address patients with associated symptoms, such as facial pain / pressure, sleep disorders, smell disorders and odontogenic problems, many of which are principal symptoms in the diagnosis of rhinosinusitis.

\section{Stakeholder involvement}

The EPOS2020 guideline development group is made up of international ENT / rhinology specialists representing a range of geographical locations and specialties, as well as other experts interested in the field (including allergologists, pulmonologists, GPs, pharmacists, paediatricians, microbiologists) and patient representatives. The group will also include key methodology 
experts. For a full list of members see Appendix 1.

Our primary target users will be ENT / rhinology specialists, providing them with an up-to-date review of the latest research and clear diagnostic and treatment strategies and recommendations. For non-ENT primary practitioners, such as GPs (including family and emergency physicians), pharmacists and paediatricians our aim is to inform them of the latest research and recommendations and provide clear care pathways. Other specialists including allergologists / immunologists, pulmonologists, doctors of internal medicine and secondary practitioners such as nurses will be provided with clear, unambiguous definitions of the terminology and information on the latest research and recommendations. Patient involvement will be a defining element of EPOS2020, and this is reflected by the inclusion of key patient representatives in the development group, along with a dissemination strategy which will target users beyond the immediate clinical sphere. The initiatives started within EUFOREA that will find a solid base in EPOS2020 and will help to better inform patients and further empower them to seek optimal help in the management of their disease ${ }^{(32-34)}$. The position paper will provide guidelines for relevant government bodies, national and international drug agencies [e.g. the European Medicines Agency (EMA) and the Food and Drug Administration (FDA) in the USA] and scientific societies, particularly regarding the use of terminology, definitions and classification for clinical trials. In addition, EPOS2020 will be an important tool for insurance companies and payers, and will act as a key reference tool for national and international mass media providing disease information to the general population.

\section{Rigour of development}

EPOS2020 will use mixed methodologies to incorporate grades of evidence and grades of expert opinion / common consensus. The aim is to combine expert opinion with methodological back-up, to be as inclusive as possible and to be clear in our definitions.

\section{Methodology}

We will use evidence-based medicine (EBM) methodologies to search for our evidence, incorporating randomized controlled trials (RCTs) with 'Real World Experience (RWE) - observational studies (surgery) / real-life studies, and using network analysis and meta-analysis of systematic reviews to combine results from clinical trials.

For all topics we will search MEDLINE (either via OVID or via PubMed) and EMBASE (via OVID or embase.com). In case of (controlled) trials we will also search the Cochrane Central Register of Controlled Trials, the Cochrane Database of Systemic Reviews, and clinical trial registers including www.clinicaltrials.gov, the
Table 2. Key considerations for EPOS2020 literature review.

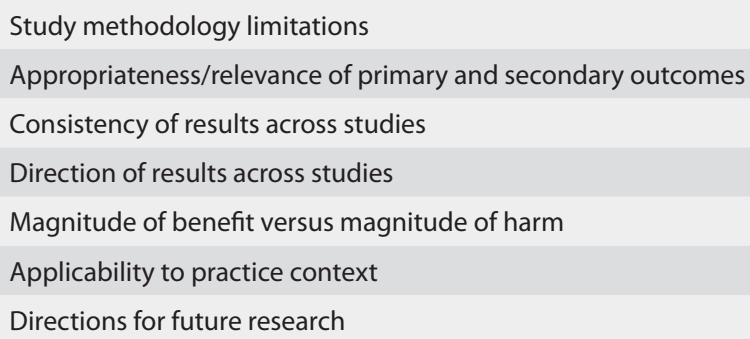

Taken from the AGREE II guidelines ${ }^{(27)}$.

WHO International Clinical Trials Registry Platform (ICTRP) and www.trialregistration.org, Depending on the topic and /or the available evidence we will also search Web of Science, Google Scholar and Google. There will be no language or date restriction. Complete search strategies will be shown in appendices to the final document.

A drawback of EBM methodology is that it relies heavily on RCTs, which are a limited way of looking at the bigger picture. This methodology is also difficult to apply to certain areas of research for EPOS2020, for example when exploring the role of surgery in rhinosinusitis. Most surgical evidence is based on observational studies, with recent research showing some justification for incorporating non-randomized studies ${ }^{(35,36)}$.

Delphi trials will be directed to ENT / rhinology specialists / primary practitioners to reach expert consensus on areas including (but not limited to) diagnostics, certain treatments for acute rhinosinusitis, and in CRS, appropriate medical therapy and surgery (and technique to use) in adult and paediatric rhinosinusitis, as well as adjunctive techniques such as image guidance. Inclusion and exclusion criteria for selecting the evidence will differ for each section of the EPOS2020 guideline. When enough RCTs with sufficient patient numbers are available, other evidence will not primarily be included. In all other situations all forms of evidence, case control, cohort studies, real-life studies and Delphi rounds will be used. GRADE (Grading of Recommendations Assessment, Development and Evaluation, http://www. gradeworkinggroup.org/) will be used whenever possible.

In-depth criteria for each section will be included in appendices to the final EPOS2020 document, see EPOS2012 for examples ${ }^{(19)}$. Details of the strengths and limitations of the body of evidence and the risk of bias will also be laid out in full. Table 2 shows the key factors which will be considered for all studies and reviews undertaken for EPOS2020, according to AGREE-II guidelines ${ }^{(27)}$.

\section{Recommendations}

Recommendations will be formulated based on review and analysis of the latest research into rhinosinusitis, combined with 
expert opinion. Using Delphi methodology, we will ensure that expert opinion is reached by common consensus in areas where the evidence base is limited. In this way we will be able to incorporate grade of evidence and grade of expert opinion.

During the formulation of recommendations for management (diagnosis, treatment and follow-up) of rhinosinusitis, all potential benefits, side effects and risks will be considered and clearly outlined, where necessary with supporting data. The grade of recommendation will also reflect these considerations, which was an effective way of presenting recommendations in EPOS2012.

Results of literature reviews and summaries of the relevant evidence base will be clearly presented to back up all recommendations. A complete reference list and bibliography will be provided to ensure that readers can access the evidence on which our recommendations are based, and to demonstrate the extent of our research.

\section{Review and updates}

The EPOS2020 position paper will be subject to a full external review by experts from a variety of specialties prior to publication for the purposes of quality assurance. We will take into account feedback from reviewers representing different communities in the world and different stakeholders (as mentioned above) on draft recommendations, applicability and feasibility when formulating the final document. A list of reviewers will be supplied as an appendix to the final document. A description of the methods for conducting the external review, along with recommendations from reviewers and outcomes of the review will be presented in an 'External Review' appendix.

The European Rhinologic Society has produced position papers on rhinosinusitis in 2007 and 2012 [following on from the first European Position Paper on Rhinosinusitis and Nasal Polyps (EP3OS) initiated by the European Academy of Allergology and Clinical Immunology in 2005] ${ }^{(16,18,19)}$. EPOS2020 will be the latest iteration of this. We expect to update the position paper again after another 5 to 6 year interval. However, the scope of the guideline may need to change in that time as the body of research into rhinosinusitis and its associated conditions continues to grow. With this in mind, following publication of EPOS2020, we will conduct a full review to measure uptake of our recommendations and the overall impact of the guidelines.

\section{Clarity of presentation}

All recommendations in EPOS2020 will be presented clearly. Levels of evidence will be presented in tables (as in EPOS2012) and recommended diagnosis, treatment and referral pathways will be presented in straightforward, accessible diagrams (as in
EPOS2012). We will make it clear which target users and population the recommendation is targeted at / applicable to and explain the intended outcome of the recommendation, along with any associated caveats and risks. Where evidence and results are uncertain this will be clearly explained, and relevant recommendations will be formulated accordingly.

All options for the screening, prevention and management (including treatment, diagnosis and follow-up) of rhinosinusitis will be presented clearly in EPOS2020. Recommendations on these options will be graded based on the available evidence and when evidence is limited, based on expert opinions using Delphi rounds. Each option will be considered as relevant to the most applicable target population and target user. For example, in EPOS2012 a full section of the guideline was dedicated to presenting evidence-based schemes for diagnosis and treatment of ARS and CRS in adult and paediatric populations and for ENT specialists and primary care practitioners.

Specific recommendations for diagnosis and treatment of each aspect of rhinosinusitis covered by the guideline will be summarised clearly, along with recommended areas for further research.

In addition to a section of the guideline presenting our recommendations in clear algorithms we will make several 'Pocket Guide' versions of EPOS2020 aimed at different stakeholders (otorhinolaryngologists, pharmacists, patients, primary care, other non-ENT specialists), translated into as many languages as possible. This was very successful for EPOS2007 and EPOS2012 as a way of presenting our key recommendations and management schemes in an accessible format for target users. We intend to develop this further with an interactive version of the guideline after publication ('wiki EPOS'), featuring extra links, videos, interactive flowcharts. This will be available online, and potentially as an easily accessible 'EPOS app'. We feel that an app version would be particularly relevant to our target population, as a way of empowering patients - directing people through the available technologies and treatments.

\section{Recommendations and applicability}

The main facilitators and barriers to applying the EPOS2020 guideline will be explored and reviewed, with feedback from key stakeholders. Although entitled 'European', which reflects its origin, the position paper is intended to be used around the world, and while it will not be possible to consider facilitators and barriers to using this document relevant to specific geographical locations, we aim to ensure it is applicable to a wide range of healthcare systems. We will therefore explore care pathways relevant to a broad range of geographical locations and healthcare systems. Facilitators and barriers to diagnosis and 
treatment which apply to all target users and populations, such as lack of research / definitive conclusions, direct and indirect medical costs and resource implications will be explored in full. We expect to uncover other facilitators and barriers to applying the guideline through Delphi trials, and will clearly indicate how the outcomes of these trials influenced our conclusions and recommendations.

The EPOS2020 steering group is presently working on a complete dissemination strategy. All members of the steering group have been asked to nominate important leaders in the field who we can be involved in the dissemination of the project. We want to include as many nationalities and relevant organisations as possible. International societies have been identified as an obvious route to target users. We also want to target student groups and non-specialist colleagues more specifically than may have happened with previous EPOS guidelines. Tools we hope to develop for putting the recommendations into practice include pocket guides aimed at different stakeholders, an interactive online version of the guideline, an EPOS2020 App and patient education videos. We aim to raise the profile of the EPOS guideline even further using the Internet, social media and by collaborating with governmental organisations to bring about targeted interventions with measurable outcomes. The goal is to disseminate our research so widely that target stakeholders and users will have it delivered to them, rather than needing to seek it out.

Where they are identified, and where possible, resource implications of applying the recommendations will be described. EPOS2020 will be an international position paper, so the resource implications of our key recommendations will vary for target users depending on their different geographical locations (this will be of particular consideration for developing countries). Where there is potential for our recommendations to have a significant impact on resources (good or bad), this will be clearly stated.

Management schemes will display clear criteria for diagnosis, management and referral of patients with rhinosinusitis, which will be derived from our key recommendations. For example, number, frequency and duration of symptoms. This will enable the application and use of the guideline to be monitored and evaluated.

\section{Editorial independence}

Although funded in large part by the European Rhinologic Society, Rhinology Journal, EUFOREA and the Rhinology Foundation and involving many of its members, EPOS2020 will be an international endeavour with contributions from a wide range of associated expert specialists who will analyse the latest research into rhinosinusitis and present up-to-date recommendations to all target users and populations. The views and interests of the ERS and other external sources of funding will not have any impact on our final recommendations, and this will be clearly stated in the final document.

There will be a large group of key stakeholders involved in the development of the EPOS2020 position paper. The competing interests of all guideline development group members will be recorded and any potential impact on the development of the guideline and our final recommendations will be addressed.

\section{Conclusion}

Patients with rhinosinusitis deserve the best possible management of their disease. We hope this article acts as a useful starting-off point for EPOS2020 and provides a detailed summary of our planned research strategy and methodologies. Our aim is for the next European Position Paper on Rhinosinusitis to promote better-informed decision-making by patients, members of the community, clinicians, and public health policy-makers on the management of rhinosinusitis.

\section{Acknowledgements}

With thanks to Polly Chester, Editorial Production Manager, EPOS2020 for researching and coordinating this article. We would also like to acknowledge our patient representatives for their role in this project.

\section{Authorship contribution}

Wytske Fokkens, Martin Desrosiers, Richard Harvey, Claire Hopkins, Joaquim Mullol, and Carl Philpott wrote the paper. All other authors commented and reviewed and gave their final approval.

\section{Conflict of interest}

The authors declare that they have no competing interests.

\section{References}

1. Hastan D, Fokkens WJ, Bachert C, Newson RB, Bislimovska J, Bockelbrink A, et al. Chronic rhinosinusitis in Europe--an underestimated disease. A GA(2)LEN study. Allergy. 2011;66(9):1216-23.

2. Hirsch AG, Stewart WF, Sundaresan AS, Young AJ, Kennedy TL, Scott Greene J, et al. Nasal and sinus symptoms and chronic rhinosinusitis in a population-based sample. Allergy. 2017;72(2):274-81.

3. Obaseki D, Potts J, Joos G, Baelum J, Haahtela T, Ahlstrom M, et al. The relation of airway obstruction to asthma, chronic rhinosinusitis and age: results from a population survey of adults. Allergy.
2014;69(9):1205-14.

4. Sundaresan AS, Hirsch AG, Young AJ, Pollak J, Tan BK, Schleimer RP, et al. Longitudinal Evaluation of Chronic Rhinosinusitis Symptoms in a Population-Based Sample. J Allergy Clin Immunol Pract. 2018;6(4):132735.e3.

5. Piatt JH, Jr. Intracranial suppuration compli- 
cating sinusitis among children: an epidemiological and clinical study. J Neurosurg Pediatr. 2011;7(6):567-74.

6. Jaume F, Quinto L, Alobid I, Mullol J. Overuse of diagnostic tools and medications in acute rhinosinusitis in Spain: a population-based study (the PROSINUS study) BMJ Open. 2018;8(1):e018788.

7. Ostovar A, Fokkens WJ, Vahdat K, Raeisi A, Mallahzadeh A, Farrokhi S. Epidemiology of chronic rhinosinusitis in Bushehr, southwestern region of Iran: a GA2LEN study. Rhinology. 2019; 57 (1): 43 - 48.

8. Veloso-Teles R, Cerejeira R, Roque-Farinha $R$, von Buchwald C. Higher prevalence of nasal polyposis among textile workers: an endoscopic based and controlled study. Rhinology. 2018.56 (2): 99-105.

9. Jarvis D, Newson R, Lotvall J, Hastan D, Tomassen $\mathrm{P}$, Keil T, et al. Asthma in adults and its association with chronic rhinosinusitis: the GA2LEN survey in Europe. Allergy. 2012;67(1):91-8

10. Hakansson K, Thomsen SF, Konge $L$, Mortensen J, Backer V, von Buchwald C. A comparative and descriptive study of asthma in chronic rhinosinusitis with nasal polyps. Am J Rhinol Allergy. 2014;28(5):383-7.

11. Frendo M, Hakansson K, Schwer S, Rix I, Ravn AT, Backer $V$, et al. Asthma in ear, nose, and throat primary care patients with chronic rhinosinusitis with nasal polyps. Am J Rhinol Allergy. 2016;30(3):67-71

12. Tay TR, Hew M. Comorbid "treatable traits" in difficult asthma: Current evidence and clinical evaluation. Allergy. 2018;73(7):136982.

13. Samitas K, Carter A, Kariyawasam HH, Xanthou G. Upper and lower airway remodelling mechanisms in asthma, allergic rhinitis and chronic rhinosinusitis: The one airway concept revisited. Allergy. 2018;73(5):993-1002.

14. van der Veen J, Seys SF, Timmermans M Levie P, Jorissen M, Fokkens WJ, et al. Reallife study showing uncontrolled rhinosinusitis after sinus surgery in a tertiary referral centre. Allergy. 2017;72(2):282-90

15. Fokkens W, Lund V, Bachert C, Clement $P$, Hellings $P$, Holmstrom $M$, et al. EAACl position paper on rhinosinusitis and nasal polyps executive summary. Allergy. 2005;60(5):583-601.

16. Fokkens $W$, Lund $V$, Bachert $C$, Clement $P$, Helllings $P$, Holmstrom M, et al. European position paper on rhinosinusitis and nasal polyps. Rhinology Supplement. 2005;18:187.
17. Fokkens W, Lund V, Mullol J. European position paper on rhinosinusitis and nasal polyps 2007. Rhinol Suppl. 2007;20:1-136.

18. Fokkens W, Lund V, Mullol J. EP3OS 2007 : European position paper on rhinosinusitis and nasal polyps 2007. A summary for otorhinolaryngologists. Rhinology. 2007:45(2):97-101.

19. Fokkens WJ, Lund VJ, Mullol J, Bachert C, Alobid I, Baroody F, et al. European Position Paper on Rhinosinusitis and Nasal Polyps 2012. Rhinol Suppl. 2012;23:3 p preceding table of contents, 1-298.

20. Fokkens WJ, Lund VJ, Mullol J, Bachert C, Alobid I, Baroody F, et al. EPOS 2012: European position paper on rhinosinusitis and nasal polyps 2012. A summary for otorhinolaryngologists. Rhinology. 2012:50(1):1-12

21. Hellings PW, Fokkens WJ, Bachert C, Akdis CA, Bieber T, Agache I, et al. Positioning the principles of precision medicine in care pathways for allergic rhinitis and chronic rhinosinusitis - A EUFOREA-ARIAEPOS-AIRWAYS ICP statement. Allergy. 2017;72(9):1297-305.

22. Jorgensen LC, Friis Christensen S, Cordoba Currea G, Llor C, Bjerrum L. Antibiotic prescribing in patients with acute rhinosinusitis is not in agreement with European recommendations. Scand J Prim Health Care. 2013;31(2):101-5.

23. Seresirikachorn K, Snidvongs K, Chitsuthipakorn W, Ruksakul W, Chusakul S, Kanjanaumporn J, et al. EPOS2012 has better specificity compared to IDSA2012 for diagnosing acute bacterial rhinosinusitis. Rhinology. 2018;56(3):241-4

24. Shi JB, Fu QL, Zhang H, Cheng L, Wang YJ, Zhu DD, et al. Epidemiology of chronic rhinosinusitis: results from a cross-sectional survey in seven Chinese cities. Allergy. 2015;70(5):533-9.

25. 2Sng WJ, Wang DY. Efficacy and side effects of antibiotics in the treatment of acute rhinosinusitis: a systematic review. Rhinology. 2015;53(1):3-9.

26. Wang DY, Wardani RS, Singh $K$, Thanaviratananich S, Vicente G, Xu G, et al. A survey on the management of acute rhinosinusitis among Asian physicians. Rhinology. 2011:49(3):264-71.

27. Consortium ANS. The AGREE II Instrument [Electronic version] 2007. Available from: http://www.agreetrust.org.

28. Avdeeva K, Fokkens W. Precision Medicine in Chronic Rhinosinusitis with Nasal Polyps. Current allergy and asthma reports.
2018;18(4):25

29. De Greve G, Hellings PW, Fokkens WJ, Pugin B, Steelant B, Seys SF. Endotype-driven treatment in chronic upper airway diseases. Clinical and translational allergy. 2017;7:22.

30. Yii ACA, Tay TR, Choo XN, Koh MSY, Tee AKH, Wang DY. Precision medicine in united airways disease: A "treatable traits" approach. Allergy. 2018;73(10):1964-78.

31. Tsetsos N, Goudakos JK, Daskalakis D, Konstantinidis I, Markou K. Monoclonal antibodies for the treatment of chronic rhinosinusitis with nasal polyposis: a systematic review. Rhinology. 2018;56(1):11-21.

32. Seys SF, Bousquet J, Bachert C, Fokkens WJ, Agache I, Bernal-Sprekelsen M, et al. mySinusitisCoach: patient empowerment in chronic rhinosinusitis using mobile technology. Rhinology. 2018;56(3):209-15.

33. Pugin B, et al. Launch of the Patient Advisory Board for Chronic Rhinosinusitis - A EUFOREA initiative. Rhinology. 2019; in press.

34. Sleurs K, Seys S, Bousquet J, Fokkens W, Gorris S, Pugin B, et al. Mobile health tools for the management of chronic respiratory diseases. Allergy. 2019

35. Higgins JPT, Altman DG, Sterne JAC. Chapter 8: Assessing risk of bias in included studies. In: Higgins JPT, Green S, editors. Cochrane Handbook for Systematic Reviews of Interventions Version 510. Oxford, UK: The Cochrane Collaboration: 2011.

36. Reeves BC, Deeks JJ, Higgins JPT, Wells GA. Chapter 13: Including non-randomized studies. In: Higgins JPT, Green S, editors. Cochrane Handbook for Systematic Reviews of Interventions Version 510. Oxford, UK: The Cochrane Collaboration; 2011.

\section{Wytske Fokkens}

Department of Otorhinolaryngology

Room A2-230

Amsterdam University Medical

Centres

Location AMC

1105 AZ Amsterdam

The Netherlands

E-mail:w.j.fokkens@amc.uva.nl

\section{Appendix 1. EPOS2020 Development Group}

\section{Wytse Fokkens}

Otorhinolaryngologist, Epidemiologist

Amsterdam UMC, Amsterdam, the Netherlands

\section{Isam Alobid}

Doctor, Professor Rhinology

Rhinology and Skull Base Unit, ENT Department, Hospital Clínic

de Barcelona, Universidad de Barcelona, August Pi i Sunyer

Biomedical Research Institute, Barcelona, Spain 


\section{Wilma Terezinha Anselmo-Lima}

Professor Otorhinolaryngology, Division of Otorhinolaryngology, Department of Ophthalmology, Otorhinolaryngology, Head

and Neck Surgery, Ribeirao Preto Medical School-University of Sao Paulo, Sao Paulo, Brazil

\section{Claus Bachert}

Professor, Doctor, Head Upper Airways Research Laboratory (URL), Chief of Clinics, Upper Airways Research Laboratory and ENT Department, University Hospital Ghent

Ghent, Belgium; and Division of ENT Diseases, CLINTEC, Karolinska Institute, University of Stockholm, Stockholm, Sweden

\section{Christian von Buchwald}

Professor, Department of Otolaryngology, Head and Neck Surgery and Audiology, Rigshospitalet, Copenhagen University, Hospital, Copenhagen, Denmark

\section{Anders Cervin}

The Garnett Passe and Rodney Williams Memorial Foundation Chair in Otolaryngology (Rhinology), Department of Otolaryngology, Head and Neck Surgery, Royal Brisbane and Women's Hospital; Faculty of Medicine, University of Queensland, Brisbane, Australia

\section{Noam Cohen}

Otorhinolaryngology - Head and Neck Surgery, Subspecialty - Rhinology, Perelman School of Medicine at The University of Pennsylvania, Philadelphia, PA, USA

\section{Jannis Constantinidis}

Professor and Chair, 1st Department of ORL, Head and Neck Surgery, Aristotle University, AHEPA Hospital, Thessaloniki, Greece

\section{Ludovic De Gabory}

Professor, Rhinologie, chirurgie des sinus, de la base du crâne et plastique de la face, CHU de Bordeaux, Hôpital Pellegrin, Centre F-X Michelet, Bordeaux, France

\section{Martin Desrosiers}

Clinical Professor, ORL-HNS, Université de Montréal, Montreal, Canada

\section{Richard Douglas}

Consultant Surgeon (ENT), Department of Surgery, The University of Auckland, Auckland, New Zealand

\section{Philippe Gevaert}

Professor in Rhinology and Allergology, Department of Otorhinolaryngology, Ghent University, Ghent, Belgium

\section{Richard Harvey}

Program Head and Professor, Rhinology and Skull Base, Applied Medical Research Centre, UNSW (Conjoint) and Macquarie University (Clinical), Sydney, Australia

\section{Peter Hellings}

Professor, Research Group Leader, Department of Otorhinolaryngology, Head and Neck Surgery, University Hospitals Leuven, KU Leuven, Leuven, Belgium

\section{Claire Hopkins}

Ear, Nose and Throat Department, Guys and St. Thomas' Hospital, London, United Kingdom

\section{Livije Kalogjera}

ENT Surgeon, Otorhinolaryngology Specialist, Professor of Otorhinolaryngology, Zagreb School of Medicine; University Hospital Center "Sestre milosrdnice", Zagreb, Croatia

\section{Rob Kern}

George A. Sisson Professor of Otolaryngology; Chair, Department of Otolaryngology - Head and Neck Surgery; Professor, Otolaryngology - Head and Neck Surgery, Medicine-AllergyImmunology, Northwestern University, Feinberg School of Medicine, Chicago, IL, USA

\section{Basile Nicolas Landis}

Professor of Otorhinolaryngology, Rhinology-Olfactology Unit, Otorhinolaryngology Department, University Hospital of Geneva, Geneva, Switzerland

\section{Valerie Lund}

Professor Emeritus in Rhinology; Honorary ENT Consultant Royal National Throat, Nose \& Ear Hospital, UCLH, London, UK

\section{Cem Meco}

Professor of Otorhinolaryngology, Head and Neck Surgery, Department of Otorhinolaryngology, Head and Neck Surgery, Salzburg Paracelsus Medical University, Salzburg, Austria; Department of Otorhinolaryngology, Head and Neck Surgery, Ankara University, Ankara, Turkey

\section{Joaquim Mullol}

Laboratory Head, Rhinology Unit and Smell Clinic, ENT Department, Hospital Clínic, IDIBAPS, Universitat de Barcelona, CIBERES Barcelona, Catalonia, Spain

\section{Carl Philpott}

Professor of Rhinology and Olfactology, Department of Medicine, Norwich Medical School, University of East Anglia Norwich, UK; ENT Department, James Paget University Hospital Great Yarmouth, UK 


\section{Sietze Reitsma}

ENT Surgeon, Rhinologist, Skull Base Surgeon, Amsterdam University Medical Centers, AMC, Amsterdam, the Netherlands

\section{Rodney Schlosser}

Professor and Director of Rhinology and Sinus Surgery, Department of Otolaryngology - Head and Neck Surgery, Medical University of South Carolina, Charleston, SC, USA

\section{Brent Senior}

Professor of Otolaryngology / Head and Neck Surgery and Professor of Neurosurgery; Chief of Rhinology, Allergy, and Endoscopic Skull Base Surgery, University of North Carolina School of Medicine, Chapel Hill, NC, USA

\section{Manuel Bernal Sprekelsen}

Professor, Doctor, Chair of ENT, Hospital Quironsalud

University of Valencia, Valencia, Spain

\section{Peter Valentin Tomazic}

Associate Professor, Department of Otorhinolaryngology, Head and Neck Surgery, Medical University of Graz, Graz, Austria

\section{Sanna Toppila-Salmi}

Otorhinolaryngologist, Skin and Allergy Hospital, Helsinki University Hospital and University of Helsinki, Helsinki, Finland

\section{De Yun Wang}

ENT Specialist and Research Professor, Department of Otolaryngology, Yong Loo Lin School of Medicine, National University of Singapore, Singapore

\section{Dehui Wang}

Chairman, Rhinology Division of ENT Department; Vice-Director, Eye and ENT Hospital, Fudan University, Shanghai, China

\section{Luo Zhang}

Professor, President, Chinese Society of Allergy; Director, Beijing Institute of Otolaryngology; President, Beijing TongRen Hospital Department of Otolaryngology Head and Neck Surgery, Beijing TongRen Hospital, Beijing, China

\section{ALLERGOLOGIST}

\section{Paolo Maria Matricardi}

Department of Pediatric Pneumology and Immunology

Charité - Universitätsmedizin Berlin, Berlin, Germany

\section{PULMONOLOGIST}

\section{Guy Joos}

Department of Respiratory Medicine, Ghent University Hospital Ghent, Belgium

\author{
GENERAL PRACTITIONERS \\ Janwillem Kocks \\ Professor of Inhalation Medicine, Observational Pragmatic Re- \\ search Institute, Singapore
}

\section{Dermot Ryan}

Allergy and Respiratory Research Group, Usher Institute of Population Health Sciences and Informatics, The University of Edinburgh, Edinburgh, UK; Optimum Patient Care, Cambridgeshire, UK

\section{PHARMACISTS}

\section{Anita Hafner}

Associate Professor, University of Zagreb Faculty of Pharmacy and Biochemistry, Zagreb, Croatia

\section{Olga Lourenco}

Professor of Pharmacotherapy, Faculty of Health Sciences, University of Beira Interior, Covilhã, Portugal

\section{MICROBIOLOGIST / INFECTIOUS DISEASES \\ Liam O'Mahony}

Professor of Medicine, Departments of Medicine and Microbiology, APC Microbiome Ireland, National University of Ireland, Cork, Ireland

\section{Sarah Lebeer}

Department of Bioscience Engineering, University of Antwerp, Antwerp, Belgium

\section{PATIENT ADVISOR}

\section{Thijs Teeling}

Task Force Healthcare, WTC Den Haag, The Netherlands

\section{Andrew Knill}

Opus Communications, London, UK

\section{PAEDIATRIC OTOLARYNGOLOGIST \\ Fuad Baroody}

Professor of Surgery (Otolaryngology-Head and Neck Surgery) and Paediatrics, The University of Chicago Medicine and the Comer Children's Hospital, Chicago, IL, USA

\section{EPIDEMIOLOGIST}

\section{Wytske Fokkens}

Otorhinolaryngologist, Epidemiologist, Amsterdam UMC, Amsterdam, the Netherlands

\section{SYSTEMATIC REVIEWS EXPERTS \\ Wytske Fokkens}

Otorhinolaryngologist, Epidemiologist, Amsterdam UMC, Am- 
sterdam, the Netherlands

\section{Richard Harvey}

Program Head and Professor, Rhinology and Skull Base, Applied Medical Research Centre, UNSW (Conjoint) and Macquarie University (Clinical), Sydney, Australia

\section{Claire Hopkins}

Ear, Nose and Throat Department, Guys and St. Thomas' Hospital, London, United Kingdom

\section{Carl Philpott}

Professor of Rhinology \& Olfactology, Norwich Medical School, University of East Anglia, Norwich, UK
MEDICAL LIBRARIAN

\section{Jacqueline Limpens}

Amsterdam UMC, Amsterdam, the Netherlands

Logo designed by Benoit Pugin.

*Please note, we are actively recruiting new members to represent patients, health economists and methodology (GRADE) expertise. 